\title{
Initial coefficient bounds for a subclass of $m$-fold symmetric bi-univalent functions
}

\author{
H. M. Srivastava ${ }^{1, *}$, S. Sivasubramanian ${ }^{2}$ and R. Sivakumar ${ }^{2}$ \\ ${ }^{1}$ Department of Mathematics and Statistics, University of Victoria, Victoria, British Columbia V8W 3R4, Canada \\ 2 Department of Mathematics, University College of Engineering, Anna University (Chennai), Tindivanam 604001, \\ Tamil Nadu, India \\ * Corresponding Author \\ E-mail: harimsri@math.uvic.ca, sivasaisastha@rediffmail.com, mrsiva75@gmail.com
}

\begin{abstract}
Let $\Sigma$ denote the class of functions

$$
f(z)=z+\sum_{n=2}^{\infty} a_{n} z^{n}
$$

belonging to the normalized analytic function class $\mathcal{A}$ in the open unit disk $\mathbb{U}$, which are bi-univalent in $\mathbb{U}$, that is, both the function $f$ and its inverse $f^{-1}$ are univalent in $\mathbb{U}$. The usual method for computation of the coefficients of the inverse function $f^{-1}(z)$ by means of the relation $f^{-1}(f(z))=z$ is too difficult to apply in the case of $m$-fold symmetric analytic functions in $\mathbb{U}$. Here, in our present investigation, we aim at overcoming this difficulty by using a general formula to compute the coefficients of $f^{-1}(z)$ in conjunction with the residue calculus. As an application, we introduce two new subclasses of the bi-univalent function class $\Sigma$ in which both $f(z)$ and $f^{-1}(z)$ are $m$-fold symmetric analytic functions with their derivatives in the class $\mathcal{P}$ of analytic functions with positive real part in $\mathbb{U}$. For functions in each of the subclasses introduced in this paper, we obtain the coefficient bounds for $\left|a_{m+1}\right|$ and $\left|a_{2 m+1}\right|$.
\end{abstract}

2010 Mathematics Subject Classification. 30C45. 30C50, 30C80

Keywords. Analytic function, Univalent functions, Bi-Univalent functions, $m$-Fold symmetric functions, $m$-Fold symmetric bi-univalent functions.

\section{Introduction and definitions}

Let $\mathcal{A}$ denote the class of functions of the following form:

$$
f(z)=z+\sum_{n=2}^{\infty} a_{n} z^{n},
$$

which are analytic in the open unit disk

$$
\mathbb{U}=\{z: z \in \mathbb{C} \text { and }|z|<1\} .
$$

We denote by $\mathcal{S}$ the class of all functions in $\mathcal{A}$ which are univalent in $\mathbb{U}$ (see, for details, [2, 4]).

It is well known that every function $f \in \mathcal{S}$ has an inverse $f^{-1}$, which is defined by

$$
f^{-1}(f(z))=z \quad(z \in \mathbb{U})
$$

Tbilisi Mathematical Journal 7(2) (2014), pp. 1-10.

Tbilisi Centre for Mathematical Sciences.

Received by the editors: 09 June 2014.

Accepted for publication: 13 October 2014 
and

$$
f\left(f^{-1}(w)\right)=w \quad\left(|w|<r_{0}(f) ; r_{0}(f) \geqq \frac{1}{4}\right) .
$$

In fact, the inverse function $f^{-1}$ may be analytically continued to $\mathbb{U}$ as follows:

$$
f^{-1}(w)=w-a_{2} w^{2}+\left(2 a_{2}^{2}-a_{3}\right) w^{3}-\left(5 a_{2}^{3}-5 a_{2} a_{3}+a_{4}\right) w^{4}+\cdots .
$$

A function $f \in \mathcal{A}$ is said to be bi-univalent in $\mathbb{U}$ if both $f$ and $f^{-1}$ are univalent in $\mathbb{U}$. Let $\Sigma$ denote the class of all functions $f \in \mathcal{A}$ which are bi-univalent in $\mathbb{U}$ and are given by the equation (1.1).

Lewin [8] investigated the class $\Sigma$ of bi-univalent functions and obtained a bound given by

$$
\left|a_{2}\right| \leqq 1.51
$$

Motivated by the work of Lewin [8], Brannan and Clunie [1] conjectured that

$$
\left|a_{2}\right| \leqq \sqrt{2}
$$

Some examples of bi-univalent functions are given (see also the work of Srivastava et al. [13]):

$$
\frac{z}{1-z}, \quad \frac{1}{2} \log \left(\frac{1+z}{1-z}\right) \quad \text { and } \quad-\log (1-z) .
$$

Indeed, as pointed out by Srivastava et al. [13], the coefficient estimate problem for each of the following Taylor-Maclaurin coefficients:

$$
\left|a_{n}\right| \quad(n \in \mathbb{N} \backslash\{1,2\} ; \mathbb{N}:=\{1,2,3, \cdots\})
$$

is still open.

In recent years, the study of bi-univalent functions has gained momentum mainly due to the work of Srivastava et al. [13], which has apparently revived the subject. Motivated by their work [13], many researchers (see, for example, [3, 5, 6, 11, 13, 14, 12, 15, 16, 17, 18]; see also the various closely-related papers on the subject, which are cited in some of these works) have recently investigated several interesting subclasses of the bi-univalent function class $\Sigma$ and found non-sharp estimates on the first two Taylor-Maclaurin coefficients of functions belonging to these subclasses.

For each function $f \in \mathcal{S}$, the function

$$
h(z)=\sqrt[m]{f\left(z^{m}\right)} \quad(z \in \mathbb{U} ; m \in \mathbb{N})
$$

is univalent and maps the unit disk $\mathbb{U}$ into a region with $m$-fold symmetry. A function is said to be $m$-fold symmetric (see $[9,10]$ ) if it has the following normalized form:

$$
f(z)=z+\sum_{k=1}^{\infty} a_{m k+1} z^{m k+1} \quad(z \in \mathbb{U} ; m \in \mathbb{N}) .
$$

We denote by $\mathcal{S}_{m}$ the class of $m$-fold symmetric univalent functions in $\mathbb{U}$, which are normalized by the series expansion (1.5). In fact, the functions in the class $\mathcal{S}$ are one-fold symmetric. 
Analogous to the concept of $m$-fold symmetric univalent functions, we here introduced the concept of $m$-fold symmetric bi-univalent functions. Each function $f \in \Sigma$ generates an $m$-fold symmetric bi-univalent function for each integer $m \in \mathbb{N}$. The normalized form of $f$ is given as in (1.5) and the series expansion for $f^{-1}$ is given as follows by using Theorem (1):

$$
\begin{aligned}
g(w)=w & -a_{m+1} w^{m+1}+\left[(m+1) a_{m+1}^{2}-a_{2 m+1}\right] w^{2 m+1} \\
& -\left[\frac{1}{2}(m+1)(3 m+2) a_{m+1}^{3}-(3 m+2) a_{m+1} a_{2 m+1}+a_{3 m+1}\right] w^{3 m+1}+\cdots,
\end{aligned}
$$

where $f^{-1}=g$. We denote by $\Sigma_{m}$ the class of $m$-fold symmetric bi-univalent functions in $\mathbb{U}$. For $m=1$, the formula (1.6) coincides with the formula (1.4) of the class $\Sigma$. Some examples of $m$-fold symmetric bi-univalent functions are given as follows:

$$
\left(\frac{z^{m}}{1-z^{m}}\right)^{\frac{1}{m}}, \quad\left[\frac{1}{2} \log \left(\frac{1+z^{m}}{1-z^{m}}\right)\right]^{\frac{1}{m}} \quad \text { and } \quad\left[-\log \left(1-z^{m}\right)\right]^{\frac{1}{m}}
$$

with the corresponding inverse functions given by

$$
\left(\frac{w^{m}}{1+w^{m}}\right)^{\frac{1}{m}},\left(\frac{e^{2 w^{m}}-1}{e^{2 w^{m}}+1}\right)^{\frac{1}{m}} \quad \text { and } \quad\left(\frac{e^{w^{m}}-1}{e^{w^{m}}}\right)^{\frac{1}{m}}
$$

respectively.

Finally, we denote by $\mathcal{P}$ the Carathéodary class of functions $p(z)$ of the following form:

$$
p(z)=1+p_{1} z+p_{2} z^{2}+\cdots,
$$

which are analytic in $\mathbb{U}$ such that

$$
\Re\{p(z)\}>0 \quad(z \in \mathbb{U}) .
$$

The objective of the present paper is to introduce an elegant formula for computing the coefficients of the inverse functions for the class $\Sigma_{m}$ of $m$-fold symmetric functions by means of residue calculus. As an application, we introduce two new subclasses of bi-univalent functions in which both $f$ and $f^{-1}$ are $m$-fold symmetric analytic functions with their derivative in the class $\mathcal{P}$ and obtain coefficient bounds for $\left|a_{m+1}\right|$ and $\left|a_{2 m+1}\right|$ for functions in each of these new subclasses.

In order to derive our main results, we use the following lemma for the Carathéodary class $\mathcal{P}$.

Lemma. If $h \in \mathcal{P}$, then

$$
\left|c_{k}\right| \leqq 2 \quad(k \in \mathbb{N})
$$

where the Carathéodary class $\mathcal{P}$ is the family of all functions $h$, analytic in $\mathbb{U}$, for which

$$
\Re\{h(z)\}>0 \quad(z \in \mathbb{U})
$$

and

$$
h(z)=1+c_{1} z+c_{2} z^{2}+\cdots \quad(z \in \mathbb{U}),
$$

the extremal function being given by

$$
h(z)=\frac{1+z}{1-z} \quad(z \in \mathbb{U}) .
$$




\section{Coefficients of the inverse functions}

Coefficients of the inverse $f^{-1}$ of a given function $f$ are obtained generally by virtue of the relation

$$
f^{-1}(f(z))=z .
$$

However, this technique is much too difficult to apply in the case of $m$-fold symmetric functions. To overcome this difficulty, we use a general formula [4] to compute the coefficients of $f^{-1}$ by means of the residue calculus as follows.

Theorem 1. Let $f(z)$ be in the class $\mathcal{S}$. Then the coefficients $\gamma_{n}$ of the inverse function

$$
f^{-1}(w)=w+\gamma_{2} w^{2}+\gamma_{3} w^{3}+\cdots
$$

are given by

$$
\gamma_{n}=\frac{1}{n !} \lim _{z \rightarrow 0}\left\{\frac{d^{n-1}}{d z^{n-1}}\left(\frac{z}{f(z)}\right)^{n}\right\} .
$$

Proof. Let $f(z)$, given by (1.4), be in the class $\mathcal{S}$ and suppose that

$$
g(w)=f^{-1}(w)
$$

is given as in the hypothesis of Theorem 1. Then, from Cauchy's integral formula and by the method described in [7], we have

$$
\gamma_{n}=\frac{1}{2 \pi i} \int_{\mathcal{C}_{\varepsilon}}\left(\frac{g(w)}{w^{n+1}}\right) d w=\frac{1}{2 n \pi i} \int_{|z|=r} \frac{d z}{[f(z)]^{n}} \quad(0<r<1),
$$

where $\mathcal{C}_{\varepsilon}$ denotes a closed positively-oriented circle in the complex $w$-plane with centre at $w=0$ and radius $\varepsilon(0<\varepsilon<1)$. Since the function $f(z)$ is univalent and $f(0)=0$, the integrand $\frac{1}{[f(z)]^{n}}$ has a pole of order $n$ at $z=0$. Therefore, we have

$$
\gamma_{n}=\frac{1}{n !} \lim _{z \rightarrow 0}\left\{\frac{d^{n-1}}{d z^{n-1}}\left(\frac{z}{f(z)}\right)^{n}\right\}
$$

which evidently proves Theorem 1 .

Q.E.D.

In view of Theorem 1, the first two coefficients $\gamma_{m+1}$ and $\gamma_{2 m+1}$ of the $m$-fold symmetric bi-univalent function $f(z)$ in (1.5) are given by

$$
\gamma_{m+1}=\frac{1}{(m+1) !} \lim _{z \rightarrow 0}\left\{\frac{d^{m}}{d z^{m}}\left(\frac{z}{f(z)}\right)^{m+1}\right\}
$$

and

$$
\gamma_{2 m+1}=\frac{1}{(2 m+1) !} \lim _{z \rightarrow 0}\left\{\frac{d^{2 m}}{d z^{2 m}}\left(\frac{z}{f(z)}\right)^{2 m+1}\right\} .
$$




\section{Coefficient bounds for the function class $\mathcal{H}_{\Sigma, m}^{\alpha}$}

Definition 1. A function $f(z)$, given by (1.5), is said to be in the class $\mathcal{H}_{\Sigma, m}^{\alpha}$ if the following conditions are satisfied:

$$
f \in \Sigma_{m} \quad \text { and } \quad\left|\arg \left\{f^{\prime}(z)\right\}\right|<\frac{\alpha \pi}{2} \quad(z \in \mathbb{U} ; 0<\alpha \leqq 1)
$$

and

$$
\left|\arg \left\{g^{\prime}(w)\right\}\right|<\frac{\alpha \pi}{2} \quad(w \in \mathbb{U} ; 0<\alpha \leqq 1),
$$

where the function $g(w)$ is given by (1.6).

In this section, we first state and prove the following theorem.

Theorem 2. Let the function $f(z)$, given by (1.5), be in the class $\mathcal{H}_{\Sigma, m}^{\alpha}(0<\alpha \leqq 1)$. Then

$$
\left|a_{m+1}\right| \leqq \frac{2 \alpha}{m \sqrt{(m+1)(\alpha m+m+1}}
$$

and

$$
\left|a_{2 m+1}\right| \leqq \frac{2 \alpha[(2 m+1) \alpha+m+1]}{(m+1)(2 m+1)} .
$$

Proof. From (3.1) and (3.2), we get

$$
f^{\prime}(z)=[p(z)]^{\alpha}
$$

and

$$
g^{\prime}(w)=[q(w)]^{\alpha},
$$

where the functions $p(z)$ and $q(w)$ are in the class $\mathcal{P}$ and have the following series representations:

$$
p(z)=1+p_{m} z^{m}+p_{2 m} z^{2 m}+p_{3 m} z^{3 m}+\cdots
$$

and

$$
q(w)=1+q_{m} w^{m}+q_{2 m} w^{2 m}+q_{3 m} w^{3 m}+\cdots .
$$

Now, equating the coefficients in (3.5) and (3.6), we find that

$$
\begin{gathered}
(m+1) a_{m+1}=\alpha p_{m}, \\
(2 m+1) a_{2 m+1}=\alpha p_{2 m}+\frac{\alpha(\alpha-1)}{2} p_{m}^{2}, \\
-(m+1) a_{m+1}=\alpha q_{m}
\end{gathered}
$$


and

$$
(2 m+1)\left[(m+1) a_{m+1}^{2}-a_{2 m+1}\right]=\alpha q_{2 m}+\frac{\alpha(\alpha-1)}{2} q_{m}^{2} .
$$

From (3.9) and (3.11), we get

$$
p_{m}=-q_{m}
$$

and

$$
2(m+1)^{2} a_{m+1}^{2}=\alpha^{2}\left(p_{m}^{2}+q_{m}^{2}\right) .
$$

Also, from (3.10),(3.12) and (3.14), a simple computation shows that

$$
\begin{aligned}
(2 m+1)(m+1) a_{m+1}^{2} & =\alpha\left(p_{2 m}+q_{2 m}\right)+\frac{\alpha(\alpha-1)}{2}\left(p_{m}^{2}+q_{m}^{2}\right) \\
& =\alpha\left(p_{2 m}+q_{2 m}\right)+\frac{\alpha(\alpha-1)}{2} \frac{2(m+1)^{2}}{\alpha^{2}} a_{m+1}^{2} .
\end{aligned}
$$

Therefore, we have

$$
a_{m+1}^{2}=\frac{\alpha^{2}\left(p_{2 m}+q_{2 m}\right)}{(m+1)(\alpha m+m+1)} .
$$

Applying the Lemma of the preceding section for the coefficients $p_{2 m}$ and $q_{2 m}$, we obtain

$$
\left|a_{m+1}\right| \leqq \frac{2 \alpha}{\sqrt{(m+1)(\alpha m+m+1)}} .
$$

This gives the desired estimate for $\left|a_{m+1}\right|$ as asserted in (3.3).

Next, in order to find the bound on $\left|a_{2 m+1}\right|$, by subtracting (3.12) from (3.10), we get

$$
\begin{aligned}
2(2 m+1) & a_{2 m+1}-(m+1)(2 m+1) a_{m+1}^{2} \\
& =\alpha\left(p_{2 m}-q_{2 m}\right)+\frac{\alpha(\alpha-1)}{2}\left(p_{m}^{2}-q_{m}^{2}\right) .
\end{aligned}
$$

By a simple computation, and using (3.13) to (3.15), we get

$$
a_{2 m+1}=\frac{\alpha^{2}\left(p_{m}^{2}+q_{m}^{2}\right)}{4(m+1)}+\frac{\alpha\left(p_{2 m}-q_{2 m}\right)}{2(2 m+1)} .
$$

Thus, by applying the Lemma of Section 1 again for the coefficients $p_{m}, p_{2 m}, q_{m}$ and $q_{2 m}$, we find that

$$
\left|a_{2 m+1}\right| \leqq \frac{2 \alpha[(2 m+1) \alpha+m+1]}{(m+1)(2 m+1)} .
$$

This completes the proof of Theorem 2 .

Q.E.D. 


\section{Coefficient bounds for the function class $\mathcal{H}_{\Sigma, m}(\beta)$}

Definition 2. A function $f(z)$, given by (1.5), is said to be in the class $\mathcal{H}_{\Sigma, m}(\beta)$ if the following conditions are satisfied:

$$
f \in \Sigma_{m} \quad \text { and } \quad \Re\left\{f^{\prime}(z)\right\}>\beta \quad(z \in \mathbb{U} ; 0 \leqq \beta<1)
$$

and

$$
\Re\left\{g^{\prime}(w)\right\}>\beta \quad(z \in \mathbb{U} ; 0 \leqq \beta<1),
$$

where the function $g$ is defined by (1.6).

In this section, we state and prove the following theorem.

Theorem 3. Let the function $f(z)$, given by (1.5), be in the class $\mathcal{H}_{\Sigma, m}(\beta) \quad(0 \leqq \beta<1)$. Then

$$
\left|a_{m+1}\right| \leqq 2 \sqrt{\frac{(1-\beta)}{(m+1)(2 m+1)}}
$$

and

$$
\left|a_{2 m+1}\right| \leqq 2(1-\beta)\left(\frac{(1-\beta)(2 m+1)+m+1}{(m+1)(2 m+1)}\right) .
$$

Proof. First of all, the argument inequalities in (4.1) and (4.2) can be written in the following forms:

$$
f^{\prime}(z)=\beta+(1-\beta) p(z)
$$

and

$$
g^{\prime}(w)=\beta+(1-\beta) q(w),
$$

where the functions $p(z)$ and $q(w)$ are in the class $\mathcal{P}$ and have the forms given by (3.7) and (3.8), respectively. Now, as in the proof of Theorem 2, by equating the coefficients in (4.5) and (4.6), we get

$$
\begin{gathered}
(m+1) a_{m+1}=(1-\beta) p_{m}, \\
(2 m+1) a_{2 m+1}=(1-\beta) p_{2 m}, \\
-(m+1) a_{m+1}=(1-\beta) q_{m}
\end{gathered}
$$

and

$$
(2 m+1)\left[(m+1) a_{m+1}^{2}-a_{2 m+1}\right]=(1-\beta) q_{2 m} .
$$

From (4.7) and (4.9), we get

$$
p_{m}=-q_{m}
$$

and

$$
2(m+1)^{2} a_{m+1}^{2}=(1-\beta)^{2}\left(p_{m}^{2}+q_{m}^{2}\right) .
$$


Also, from (4.8) and (4.10), we obtain

$$
(m+1)(2 m+1) a_{m+1}^{2}=(1-\beta)\left(p_{2 m}+q_{2 m}\right) .
$$

Thus, clearly, we have

$$
\left|a_{m+1}\right|^{2} \leqq \frac{(1-\beta)\left(\left|p_{2 m}\right|+\left|q_{2 m}\right|\right)}{(m+1)(2 m+1)} \leqq \frac{4(1-\beta)}{(m+1)(2 m+1)} .
$$

This gives the bound on $\left|a_{m+1}\right|$ as asserted in (4.3).

Next, in order to find the bound on $\left|a_{2 m+1}\right|$, by subtracting (4.10) from (4.8), we get

$$
2(2 m+1) a_{2 m+1}-(m+1)(2 m+1) a_{m+1}^{2}=(1-\beta)\left(p_{2 m}-q_{2 m}\right)
$$

or, equivalently,

$$
a_{2 m+1}=\frac{(m+1)}{2} a_{m+1}^{2}+\frac{(1-\beta)\left(p_{2 m}-q_{2 m}\right)}{2(2 m+1)} .
$$

Upon substituting the value of $a_{m+1}^{2}$ from (4.12), we get

$$
a_{2 m+1}=\frac{(1-\beta)^{2}\left(p_{m}^{2}+q_{m}^{2}\right)}{4(m+1)}+\frac{(1-\beta)\left(p_{2 m}-q_{2 m}\right)}{2(2 m+1)} .
$$

Applying the Lemma of Section 1 for the coefficients $p_{m}, p_{2 m}, q_{m}$ and $q_{2 m}$, we find that

$$
\left|a_{2 m+1}\right| \leqq 2(1-\beta)\left(\frac{(1-\beta)(2 m+1)+m+1}{(m+1)(2 m+1)}\right)
$$

which is the bound on $\left|a_{3}\right|$ as asserted in (4.4).

Q.E.D.

\section{Corollaries and consequences}

For one-fold symmetric bi-univalent functions, Theorem 2 and Theorem 3 reduce to Corollary 1 and Corollary 2, respectively, which were proven earlier by Srivastava et al. [13].

Corollary 1. (see [13]) Let the function $f(z)$, given by $(1.1)$, be in the class $\mathcal{H}_{\Sigma}^{\alpha}(0<\alpha \leqq 1)$. Then

$$
\left|a_{2}\right| \leqq \alpha \sqrt{\frac{2}{2+\alpha}}
$$

and

$$
\left|a_{3}\right| \leqq \frac{\alpha(3 \alpha+2)}{3}
$$

Corollary 2. (see [13]) Let the function $f(z)$, given by $(1.1)$, be in the class $\mathcal{H}_{\Sigma}(\beta) \quad(0 \leqq \beta<1)$. Then

$$
\left|a_{2}\right| \leqq \sqrt{\frac{2(1-\beta)}{3}}
$$

and

$$
\left|a_{3}\right| \leqq \frac{(1-\beta)(5-3 \beta)}{3} .
$$

Numerous other (presumably new) corollaries and consequences of our main results can also be deduced. 


\section{References}

[1] D. A. Brannan and J. G. Clunie (Editors), Aspects of contemporary complex analysis (Proceedings of the NATO Advanced Study Institute held at the University of Durham, Durham; July 1-20, 1979), Academic Press, New York and London, 1980.

[2] P. L. Duren, Univalent functions, Grundlehren der Mathematischen Wissenschaften, Band 259, Springer-Verlag, New York, Berlin, Heidelberg and Tokyo, 1983.

[3] B. A. Frasin and M. K. Aouf, New subclasses of bi-univalent functions, Appl. Math. Lett. 24 (2011), 1569-1573.

[4] A. W. Goodman, Univalent functions, Vols. 1 and 2, Mariner Publishing Company, Tampa, Florida, 1983.

[5] S. P. Goyal and P. Goswami, Estimate for initial Maclaurin coefficients of bi-univalent functions for a class defined by fractional derivatives, J. Egyptian Math. Soc. 20 (2012), 179-182.

[6] T. Hayami and S. Owa, Coefficient bounds for bi-univalent functions, Pan Amer. Math. J. 22 (4) (2012), 15-26.

[7] J. G. Krzyż, R. J. Libera and E. J. Złotkiewicz, Coefficients of inverses of regular starlike functions, Ann. Univ. Mariae Curie-Skłodowska Sect. A 33 (1979), 103-110.

[8] M. Lewin, On a coefficient problem for bi-univalent functions, Proc. Amer. Math. Soc. 18 (1967), 63-68.

[9] W. Koepf, Coefficients of symmetric functions of bounded boundary rotations, Proc. Amer. Math. Soc. 105 (1989), 324-329.

[10] C. Pommerenke, On the coefficients of close-to-convex functions, Michigan Math. J. 9 (1962), 259-269.

[11] H. M. Srivastava, Some inequalities and other results associated with certain subclasses of univalent and bi-univalent analytic functions, in Nonlinear Analysis: Stability, Approximation, and Inequalities (Panos M. Pardalos, Pando G. Georgiev and Hari M. Srivastava, Editors.), Springer Series on Optimization and Its Applications, Vol. 68, Springer-Verlag, Berlin, Heidelberg and New York, 2012, pp. 607-630.

[12] H. M. Srivastava, S. Bulut, M. Çağlar and N. Yağmur, Coefficient estimates for a general subclass of analytic and bi-univalent functions, Filomat 27 (2013), 831-842.

[13] H. M. Srivastava, A. K. Mishra and P. Gochhayat, Certain subclasses of analytic and biunivalent functions, Appl. Math. Lett. 23 (2010), 1188-1192.

[14] H. M. Srivastava, G. Murugusundaramoorthy and N. Magesh, Certain subclasses of biunivalent functions associated with the Hohlov operator, Global J. Math. Anal. 1 (2) (2013), $67-73$.

[15] Q.-H. Xu, H. M. Srivastava and Z. Li, A certain subclass of analytic and close-to-convex functions, Appl. Math. Lett. 24 (2011), 396-401. 
[16] Q.-H. Xu, Y.-C. Gui and H. M. Srivastava, Coefficient estimates for a certain subclass of analytic and bi-univalent functions, Appl. Math. Lett. 25 (2012), 990-994.

[17] Q.-H. Xu, H.-G. Xiao, and H. M. Srivastava, A certain general subclass of analytic and biunivalent functions and associated coefficient estimate problems, Appl. Math. Comput. 218 (2012), 11461-11465.

[18] Q.-H. Xu, C.-B. Lv and H. M. Srivastava, Coefficient estimates for the inverses of a certain general class of spirallike functions, Appl. Math. Comput. 219 (2013), 7000-7011. 